\title{
Amphiregulin as a Novel Resistance Factor for Amrubicin in Lung Cancer Cells
}

\author{
SHUNTARO TOKUNAGA ${ }^{1}$, TATSUYA NAGANO ${ }^{1}$, KAZUYUKI KOBAYASHI $^{1}$, \\ MASAHIRO KATSURADA ${ }^{1}$, KYOSUKE NAKATA ${ }^{1}$, MASATSUGU YAMAMOTO ${ }^{1}$, MOTOKO TACHIHARA ${ }^{1}$, \\ HIROSHI KAMIRYO $^{1}$, HIROSHI YOKOZAKI ${ }^{2}$ and YOSHIHIRO NISHIMURA ${ }^{1}$ \\ ${ }^{1}$ Division of Respiratory Medicine, Department of Internal Medicine, \\ Kobe University Graduate School of Medicine, Kobe, Japan; \\ ${ }^{2}$ Division of Pathology, Department of Pathology, \\ Kobe University Graduate School of Medicine, Kobe, Japan
}

\begin{abstract}
Background/Aim: Amrubicin (AMR) has shown promising activity for lung cancer. However, little is known about the mechanism underlying resistance to this agent. The aim of this study was to elucidate the mechanism underlying resistance to AMR. Materials and Methods: We first developed amrubicinol (AMR-OH)-resistant cell lines (H520/R and DMS53/R) by exposing lung cancer cell lines (H520 and DMS53) to increasing concentrations of AMR$\mathrm{OH}$ and performed functional analysis by using these cell lines. Results: Transcriptome analyses showed that amphiregulin (AREG) was the most highly up-regulated gene in both AMR-OH-resistant cell lines compared to parent cells. Conditioned medium from DMS53/R cells reduced the sensitivity to AMR-OH in DMS53 cells. In contrast, $D M S 53 / R$ cells transfected with siRNA directed against $A R E G$ recovered their sensitivity to $A M R-O H$. An additional administration of cetuximab with amrubicinol also restored the sensitivity to AMR-OH. Conclusion: Amphiregulin plays an important role in resistance to $A M R-O H$.
\end{abstract}

Lung cancer is a major cancer and the leading cause of death worldwide and small-cell lung cancer (SCLC) accounts for almost $13 \%$ of all new cases (1). More than half of these patients are diagnosed with extensive-disease (ED) SCLC. SCLC refers to a rapidly proliferating tumor that is highly

This article is freely accessible online.

Correspondence to: Tatsuya Nagano, MD, Ph.D., Division of Respiratory Medicine, Department of Internal Medicine, Kobe University Graduate School of Medicine, 7-5-1 Kusunoki-cho, Chuo-ku, Kobe, 650-0017, Japan. Tel: +81 783825660; Fax: +81 783825661, e-mail: tnagano@med.kobe-u.ac.jp

Key Words: Amrubicin, lung cancer, amphiregulin, cetuximab. sensitive to chemotherapy. However, the rapid emergence of clinical drug resistance has resulted in a poor prognosis with almost all such patients dead within two years of the initial diagnosis (2).

Some multidrug resistance (MDR) mechanisms of SCLC are known. For example, overexpression of outer-membrane proteins, such as P-glycoprotein (P-gp) as a member of the ATPbinding cassette (ABC) family, is related to SCLC resistance (3). Down-regulation of topoisomerase expression is another major reason for SCLC resistance (4). Overexpression of anti-apoptotic genes, like $B C L-2$, is also related to resistance (5).

Amrubicin (AMR) is a synthetic anthracycline with a structure similar to doxorubicin approved by the Japanese government in 2002. Previous studies have suggested that AMR may be a good choice for treating lung cancer, especially relapsed SCLC (6). A phase III study of AMR as a second-line treatment for SCLC showed overall response rates of approximately $30 \%$. AMR is also effective for nonSCLC (NSCLC). A phase II study of AMR showed overall response rates of approximately $20 \%$ in previously untreated NSCLC patients (7). AMR treatment is an active and welltolerated regimen in patients with previously treated NSCLC. However, despite these good results, there are few durable responses to AMR and tumors develop resistance to AMR, usually within 4 to 12 months (8).

It was reported that lung cancer cell lines resistant to amrubicinol (AMR-OH), an active metabolite of AMR, expressed the MDRl gene encoding P-gp, whose function is the energy-dependent export of substances out of cells (3). Cytotoxicity of AMR-OH in AMR-OH-resistant cells transfected with siRNA against MDRl was restored compared to the negative control siRNA (3). However, a large study, including 130 patients, reported no association between the MDR1 protein expression and the chemotherapy response rates, progression-free survival (PFS) or overall survival (OS) (9). 
Therefore, there is still a critical need for the clarification of the resistance mechanisms and the development of new treatment strategies for patients after treatment with AMR.

\section{Materials and Methods}

Cell lines and reagents. The lung cancer cell lines DMS53 (smallcell carcinoma) and H520 (squamous cell carcinoma) were obtained from American Type Culture Collection (Manassas, VA, USA). They were maintained in RPMI 1640 medium (Sigma, St. Louis, MO, USA) supplemented with $10 \%$ fetal bovine serum (Biowest, Nuaillé, France) and 1\% penicillin-streptomycin-amphotericin B solution (Wako, Osaka, Japan) in a humidified atmosphere containing $5 \% \mathrm{CO}_{2}$ at $37^{\circ} \mathrm{C}$. AMR and AMR-OH were obtained from Dainippon Sumitomo Pharmaceuticals Co., Ltd. (Osaka, Japan). Cetuximab (CET) was purchased from Merck Serono (Tokyo, Japan). Recombinant amphiregulin (AREG) (Santa Cruz Biotechnology, Dallas, CA, USA) was used.

Establishment of AMR-OH-resistant cell lines. AMR-OH-resistant cell lines (DMS53/R and H520/R) were established using a stepwise method described elsewhere (10). Lung cancer cells were cultured in the presence of AMR-OH, starting at a concentration of one-tenth of the half-maximal inhibitory concentration $\left(\mathrm{IC}_{50}\right)$, as determined by an MTT assay, and then increased incrementally to 50 -fold the $\mathrm{IC}_{50}$ over 6 months.

Enzyme-linked immunosorbent assay (ELISA) for AREG. After $72 \mathrm{~h}$ of incubation, the AREG concentrations in the sensitive cells (DMS53/S) or DMS53/R culture supernatant were determined using a Human AREG Quantikine ELISA Kit (R\&D Systems, Minneapolis, MN, USA).

Conditioned media. The conditioned media were obtained from DMS53/S or DMS53/R cell supernatants after a 72-h incubation. To determine the growth inhibitory effects of conditioned medium on cell growth, we used an MTT assay as described (11). Briefly, cells were incubated with fresh medium or conditioned medium containing various concentrations of AMR-OH for $72 \mathrm{~h}$.

RNA isolation and microarray. Total cellular RNA preparation from DMS53/S or DMS53/R was performed as described (12). Total RNA labeled with Cy3 or Cy5 was hybridized to a 3D-Gene Human Oligo chip 25 k (25,370 distinct genes; Toray Industries Inc., Tokyo, Japan). Genes with Cy3/Cy5 normalized ratios greater than 2.0 were identified.

Quantitative reverse transcription-polymerase chain reaction ( $q R T$ $P C R)$. qRT-PCR was performed using a Thermal Cycler Dice Real Time System II (Takara Bio, Kyoto, Japan). Amplifications were performed in duplicate with SYBR Premix Ex Taq (Takara) in accordance with the manufacturer's instructions. Relative mRNA levels were calculated with the $\triangle \triangle \mathrm{Ct}$ method using glyceraldehyde3-phosphate dehydrogenase (GAPDH) mRNA as an internal control. The primers used in this study are as follows: 5'-GTGGTG CTGTCGCTCTTGATACTC-3' and 5'-TCAAATCCATCAGCACT GTGGTC-3' for AREG (13), and 5'-GCACCGTCAAGGCTG AGAAC-3' and 5'-ATGGTGGTGAAGACGCCAGT-3' for GAPDH (11). The experiments were performed in triplicate.

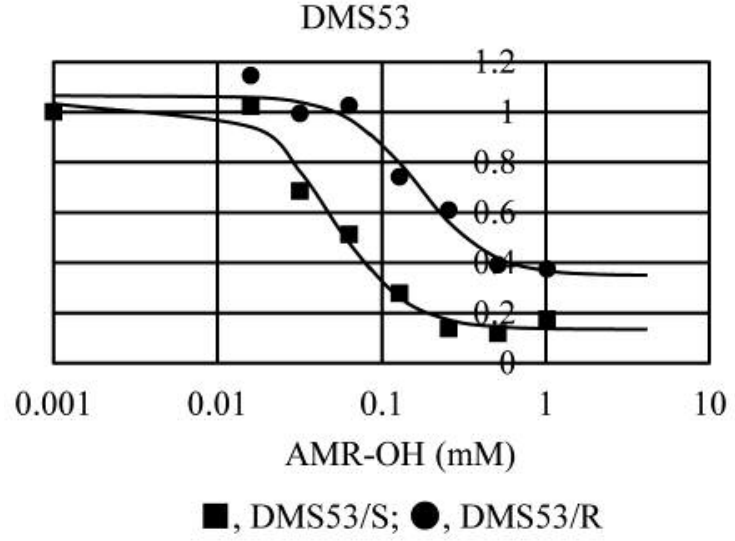

H520

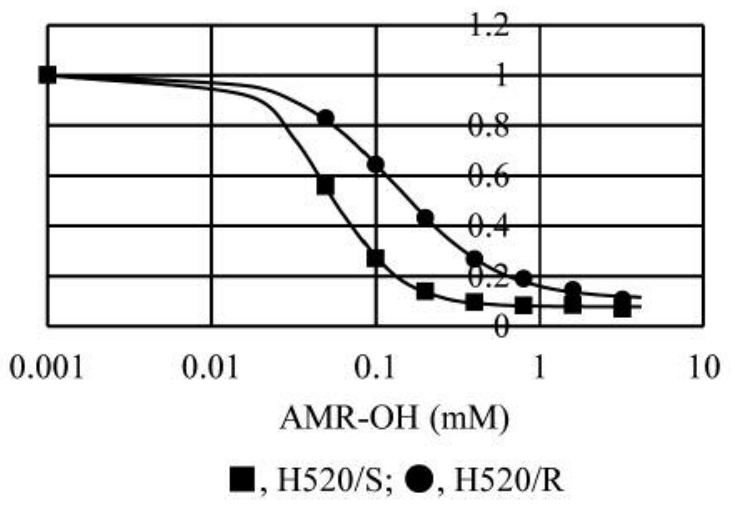

Figure 1. Two lung cancer cell lines (DMS53, H520) showed resistance to amrubicinol (AMR-OH) after a 3-month exposure of AMR-OH.

Western blot analysis. The preparation of cell lysates, sodium dodecyl sulfate-polyacrylamide gel electrophoresis (SDS-PAGE) and immunoblotting were performed as described previously (11). The primary antibodies used were $\beta$-actin (\#4967; Cell Signaling Technology Inc., Danvers, MA, USA), epidermal growth factor receptor (EGFR) (\#4267; Cell Signaling Technology), phosphoEGFR (pEGFR) (\#2234; Cell Signaling Technology), total protein kinase B (AKT) (\#4685; Cell Signaling Technology), pAKT (\#4060; Cell Signaling Technology), total extracellular signal-regulated kinase (ERK) (\#4695; Cell Signaling Technology) and pERK (\#4370; Cell Signaling Technology).

SiRNA and transfection. AREG siRNA (sc-39412) and negative control siRNA (sc37007) were purchased from Santa Cruz Biotechnology. A total of $2 \times 10^{5}$ cells were transfected with siRNA or control siRNA using the transfection reagent sc36868 (Santa Cruz Biotechnology) in serum-free RPMI-1640 medium, as described previously (11). The cells were then re-suspended in complete RPMI-1640 medium. After $48 \mathrm{~h}$ of incubation, cells were used for further experiments.

Experimental mouse models. Female BALB/c nude mice (6-weekold) were purchased from SLC Japan (Shizuoka, Japan). Mice were 
A

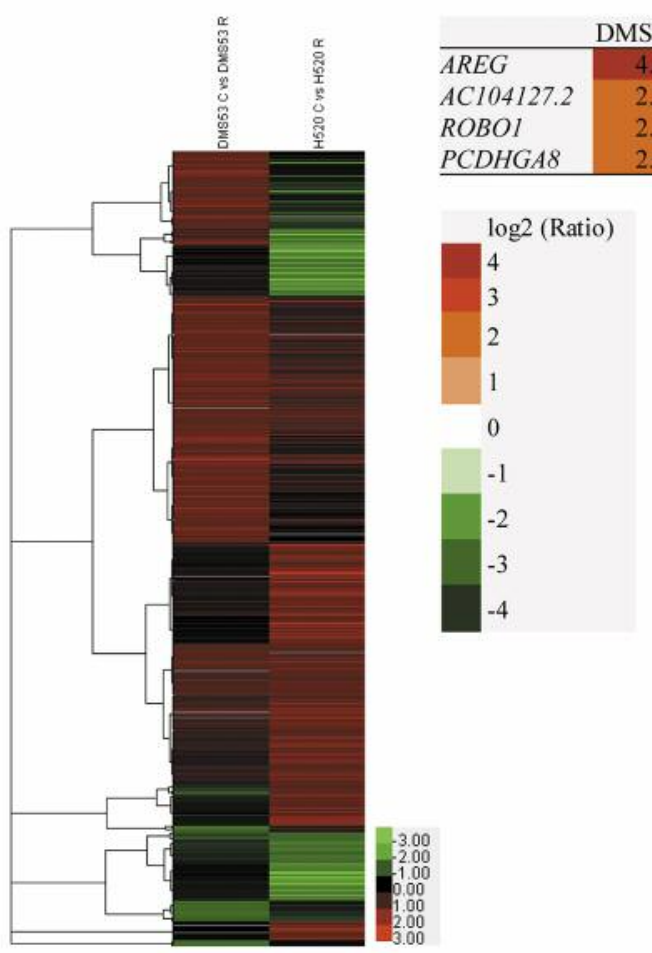

$\mathrm{C}$

\begin{tabular}{lrr} 
& DMS53 & \multicolumn{1}{c}{$\mathrm{H} 520$} \\
\hline ABCBI(MDRI) & 0.2 & \multicolumn{1}{c}{} \\
ABCB4 & 0.12 & 0.49 \\
ABCB6 & -0.71 & 0.28 \\
TAP2 & -0.3 & -1.16 \\
ABCC3 & 0.25 & 0.04 \\
ABCC8 & -0.27 & -0.38 \\
ABCA4 & -0.23 & -0.42 \\
ABCA12 & 1.57 & 0.75 \\
ABCA13 & - & 0.96 \\
GSTA2 & 0.84 & 0.6 \\
GSTA5 & 0.35 & 0.04 \\
TRAPI & -0.26 & -0.3 \\
NFKB2 & -0.47 & 0.31 \\
NFKBIL1 & -0.28 & -0.01 \\
ERCC1 & 0.1 & -0.43 \\
TP53INP1 & 0.32 & - \\
TP53INP2 & -0.01 & -0.54 \\
BIRC3 & 0.98 & 2.2 \\
\hline
\end{tabular}

$\mathrm{D}$

\begin{tabular}{lrr} 
& DMS53 & \multicolumn{1}{l}{ H520 } \\
\hline AREG & 4.03 & 2.67 \\
BTC & 0.06 & 0.36 \\
EGF & -0.43 & -0.18 \\
HB-EGF & 0.38 & -0.04 \\
TGF-alpha & 0.02 & -1.99 \\
EREG & - & - \\
& & \\
LIF & 1.67 & 1.03 \\
NRG1 & -0.07 & 1.15 \\
NRG2 & 0.17 & -0.25 \\
NRG3 & -0.03 & -0.17 \\
NRG4 & -0.17 & -0.19 \\
& & \\
HGF & 1.4 & \\
FGF-6 & 1.11 & - \\
LEP & - & 1.32 \\
TGF-betal & -0.48 & 0.52 \\
TGF-beta2 & -0.18 & 0.43 \\
TGF-beta3 & -0.06 & 0.56 \\
& & \\
ADAM10 & -0.04 & -0.19 \\
ADAM17 & 0.52 & -0.08 \\
FGF13 & & \\
\hline
\end{tabular}

Figure 2. Microarray analyses of two pairs of lung cancer cells (DMS53R/S and H520R/S) showed in increased expression of amphiregulin (AREG) through acquisition of resistance to amrubicinol (AMR-OH). (A) A hierarchical cluster analysis was performed with Pearson's correlation coefficient using log2 ratio data. A total of 2,442 genes that were up-regulated to more than 2-fold or down-regulated to less than 2-fold in at least 1 pair were analyzed. (B) Only 4 genes were up-regulated 4-fold in both pairs. AREG was the gene most up-regulated in DMS53 cells. (C) No typical drug resistance genes showed more than two-fold up-regulation or down-regulation in either cell line. (D) AREG was the only ligand of EGFR that showed more than two-fold up-regulation in both cell lines.

inoculated subcutaneously in the flank with $2 \times 10^{6}$ cells $/ 50 \mu$ single cell suspension of DMS53/R.

This study was reviewed and approved by the Institutional Animal Committee of Kobe University Graduate School of Medicine (Permit number: P15070).

In vivo growth inhibition assay. Tumor volume (TV) was measured twice a week using calipers to determine the longest (a) and shortest (b) diameters and calculated using the following equation:

$\mathrm{TV}=\left(\mathrm{a} \times \mathrm{b}^{2}\right) / 2$

When the TV reached $100 \mathrm{~mm}^{3}$, mice were divided into test groups consisting of 3 mice per group (day 0). The first group was injected intraperitoneally with CET at $100 \mu \mathrm{l} / \mathrm{animal}$ twice a week. The second group was injected with AMR at $25 \mathrm{mg} / \mathrm{kg}$ via the tail vein on day 1 . The third group of mice was treated with a combination of CET and AMR at the same dose. A control group of mice (fourth) was injected intraperitoneally with sterile PBS under similar conditions.

Statistical analyses. Data were analyzed with Student's $t$-test. If the $p$-value was smaller than 0.05 , the difference was considered to be statistically significant. All statistical tests were two-sided and data were expressed as mean \pm standard deviation $(\mathrm{SD})$.

\section{Results}

The two AMR-OH-resistant lung cancer cell lines (DMS53/R and H520/R) were established by continuously exposing each cell to AMR-OH. The $\mathrm{IC}_{50}$ values of AMR-OH for DMS53/S cells and DMS53/R cells were 0.061 and $0.322 \mu \mathrm{mol} / 1$, respectively (Figure 1A). DMS53/R cells were 5.3-fold more resistant to AMR-OH than DMS53/S cells. The $\mathrm{IC}_{50}$ values of AMR-OH for H520/S and H520/R cells were 0.052 and $1.34 \mu \mathrm{mol} / 1$, respectively (Figure $1 \mathrm{~B}$ ). H520/R cells were 2.8fold more resistant to AMR-OH than H520/S

Microarray analyses were carried out in two pairs of lung cancer cells (DMS53/S and DMR53/R or H520/S and H520/R). A hierarchical clustering analysis was performed using Pearson's correlation coefficient based on the $\log 2$ ratio data (Figure 2A). A total of 2,442 genes that were upregulated to more than 2-fold or down-regulated to less than 2 -fold in at least 1 pair were analyzed. Only 4 genes were up-regulated 4-fold in both pairs (Figure 2B). Among these genes, $A R E G$ was the gene most up-regulated in DMS53/R 
A

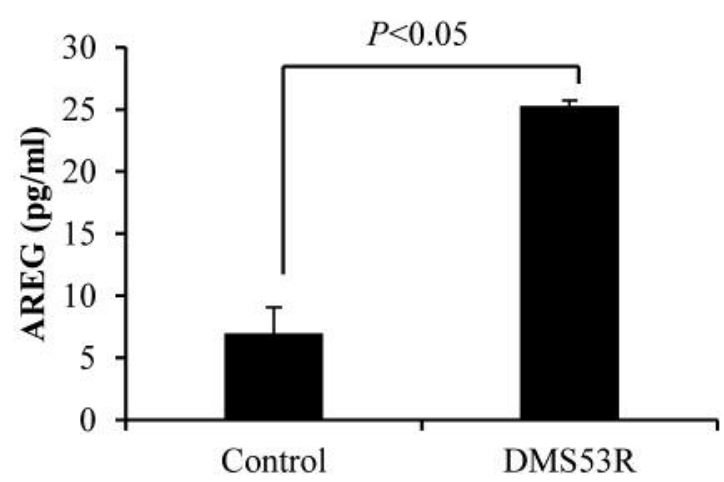

$\mathrm{C}$

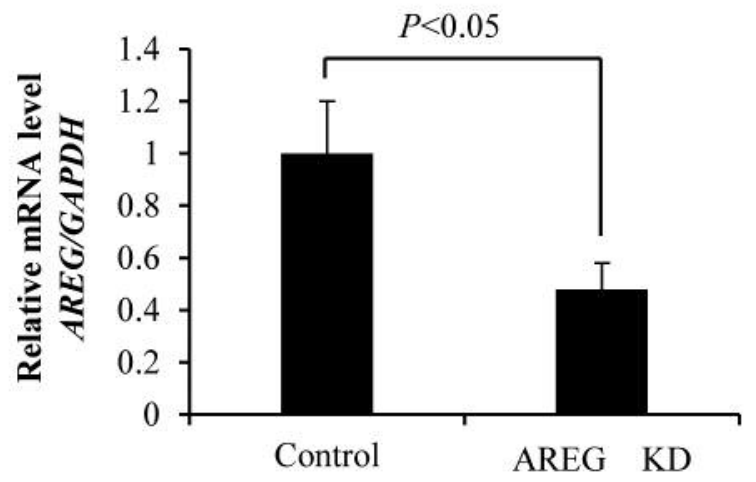

B

DMS53/S with conditioned media from control or DMS53/R

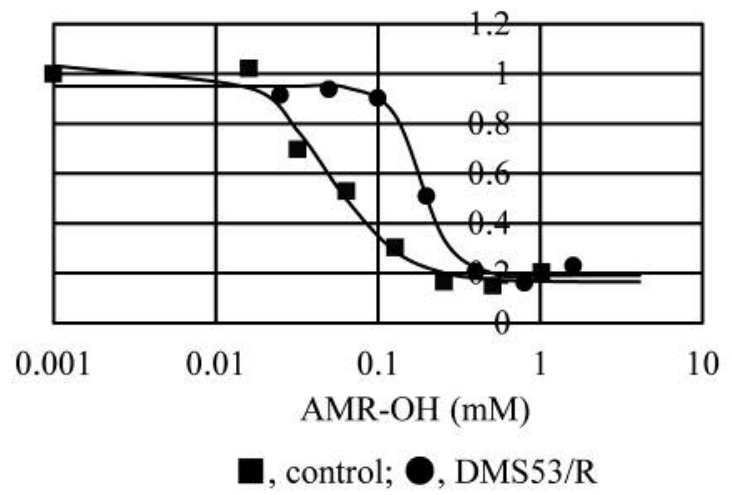

D

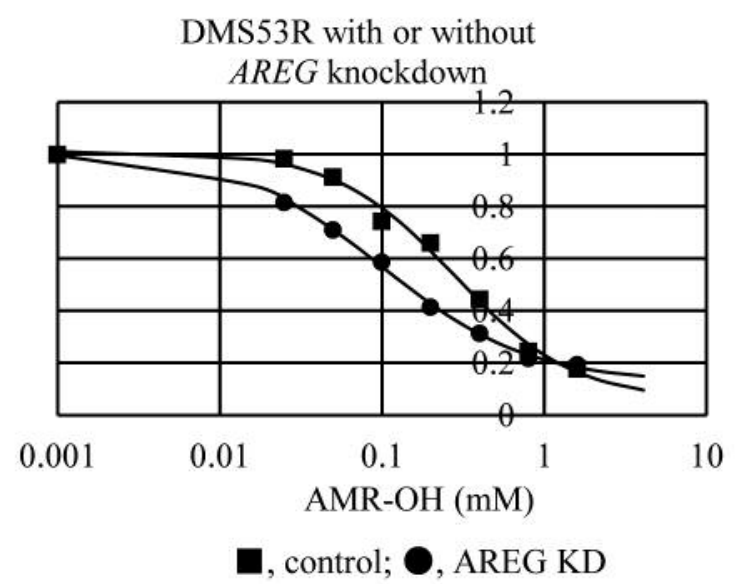

Figure 3. Amphiregulin (AREG) leads to resistance to amrubicinol (AMR-OH). (A) ELISA analysis showed that the AREG concentration in the $D M S 53 / R$ cell culture medium was 3.8-fold higher than that in the DMS53/S cell culture medium after a 72-h culture. (B) MTT assays were performed by culturing DMS53S cells in media conditioned with AMR-OH from DMS53S or DMS53R cells. Conditioned media from DMS53R cells induced resistance to AMR-OH. (C) The expression of AREG mRNA was down-regulated by siRNA against AREG. (D) The knock-down of AREG ameliorated the sensitivity to AMR-OH.

cells compared to DMS53/S cells. No previously reported drug resistance genes showed more than two-fold upregulation or down-regulation in both cell lines (Figure 2C). AREG was the only ligand of epidermal growth factor receptor (EGFR) that showed more than two-fold upregulation in either cell line (Figure 2D).

We next analyzed the role of AREG, which was released into the DMS53/R cell culture medium. At $72 \mathrm{~h}$ after incubation of DMS53/R cells, the AREG concentration of culture medium was measured by ELISA. The AREG concentration in the DMS53/R cell culture medium was 3.8fold higher than that in the DMS53/S cell culture medium (Figure $3 \mathrm{~A}$ ). The $\mathrm{IC}_{50}$ values of AMR-OH for DMS53/S cells treated with conditioned medium from DMS53/S and DMS53/R were 0.06 and $0.20 \mu \mathrm{mol} / 1$, respectively (Figure 3B). The AREG secreted by DMS53/R cells made the DMS53/S cells 3.3-fold more resistant to AMR-OH than that secreted by DMS53/S cells.

To determine the effect of $A R E G$ knockdown on the sensitivity to AMR-OH, the gene expression of $A R E G$ was knocked-down by siRNA of AREG. The efficiency of $A R E G$ suppression caused by treatment with siRNA of $A R E G$ is shown in Figure 3C. The $\mathrm{IC}_{50}$ values of $\mathrm{AMR}-\mathrm{OH}$ in AREG knockdown cells and control cells were 0.320 and 0.130 $\mu \mathrm{mol} / 1$, respectively (Figure 3D). AREG knockdown restored the sensitivity to AMR-OH. 
A

DMS53R with or without CET

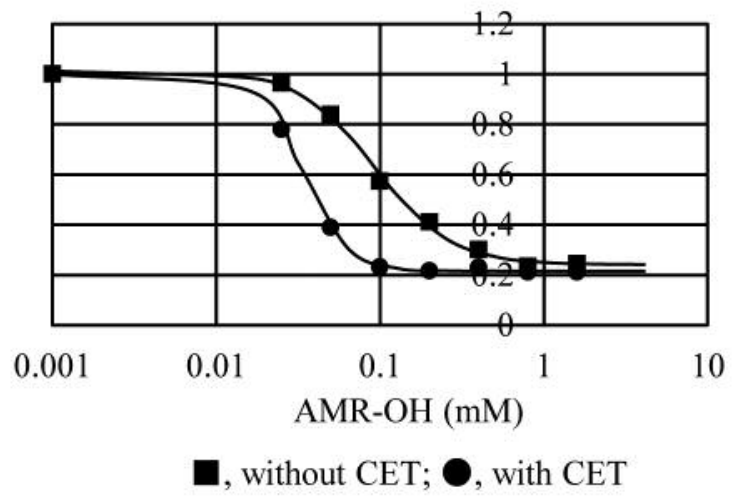

$\mathrm{C}$

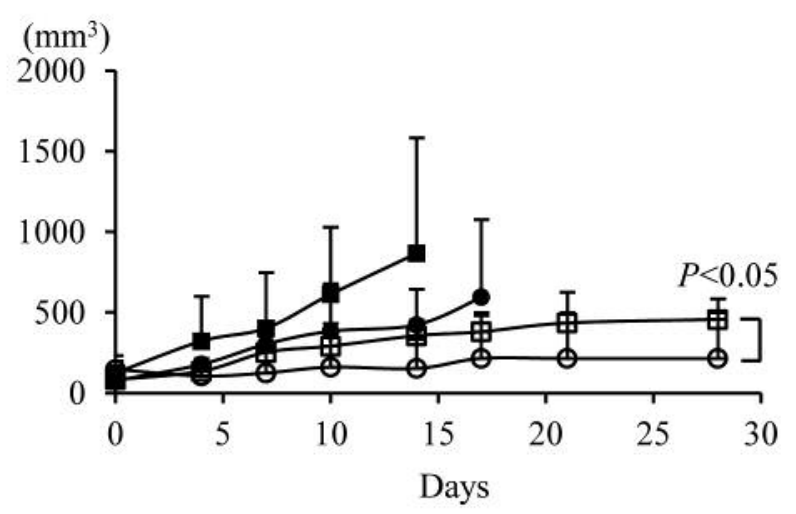

, control; O, CET; $\square$, AMR; O, AMR/CET
B

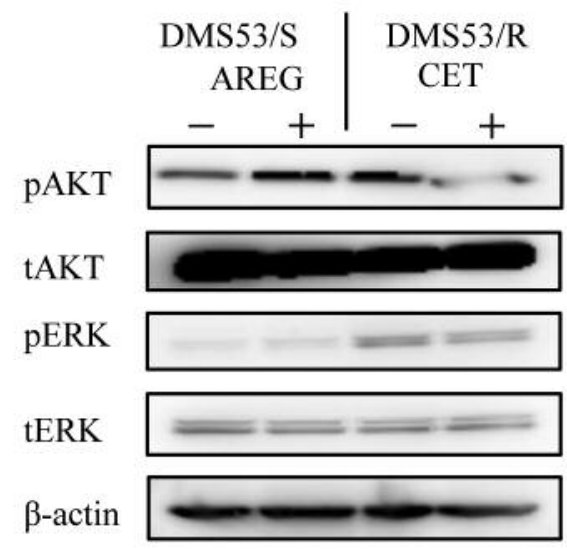

D

(g)

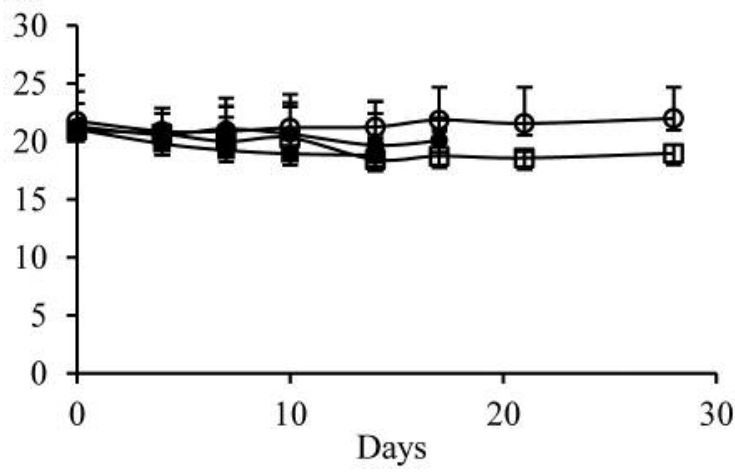

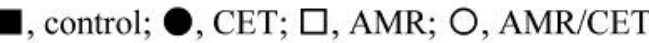

Figure 4. Cetuximab (CET) restored the sensitivity to amrubicinol (AMR-OH). (A) Combination with CET (1 $\mu$ M) restored the sensitivity to AMROH. (B) DMS53S cells were cultured with or without recombinant amphiregulin (AREG). DMS53R cells were treated with or without CET. Cell lysates were subjected to immunoblot analysis with the indicated antibodies. (C) Mice bearing a DMS53R xenograft were treated with CET (100 Ml/animal), amrubicin (AMR; $25 \mathrm{mg} / \mathrm{kg}$ ) and AMR/CET. A significant difference in the tumor volume (TV) on day 28 was found between $A M R$ - and AMR/CET-treated animals (p<0.05). (D) Treatment-related body weight $(B W)$ loss was not markedly different among the four groups.

EGFR monoclonal antibody (CET) might be useful for blocking the downstream signaling of AREG. The sensitivity of DMS53/R cells with CET $(1 \mu \mathrm{M})$ was compared to that of DMS53/R cells without CET by an MTT assay. The $\mathrm{IC}_{50}$ values of AMR-OH for DMS53/R cells with and without CET were 0.32 and $0.13 \mu \mathrm{mol} / 1$, respectively (Figure 4A). The addition of CET to AMR-OH restored the sensitivity of DMS53/R cells to AMR-OH.

DMS53/S cells were cultured with or without recombinant AREG and then treated with or without CET. Cell lysates were subjected to an immunoblot analysis with a rabbit antipEGFR, p-AKT and p-ERK1/2 antibody after $24 \mathrm{~h}$ of treatment. The baseline expression of pEGFR, p-AKT and pERK1/2 in DMS53/R cells was higher than that in DMS53/S cells (Figure 4B). CET decreased the expression of pEGFR, p-AKT and p-ERK1/2 in DMS53/R cells.

We also evaluated the efficacy and adverse effects of CET and AMR by relative tumor volume (RTV) and relative body weight (RBW), respectively. The RTV of a group treated with a combination of CET and AMR was lower than that of a group treated with AMR without any significant change in the RBW among the four groups (Figure 4C and D).

\section{Discussion}

The current study showed that AREG is induced by exposure of AMR-OH. Conditioned media from AMR-OH-resistant 
lung cancer cells induced AMR-OH-sensitive cells to acquire resistance to AMR-OH. However, AMR-OH-resistant cells transfected with siRNA directed against AREG recovered their sensitivity to AMR-OH. These results suggest that AREG plays a pivotal role in AMR-OH resistance in lung cancer cell lines.

AREG is a ligand of EGFR, a widely expressed transmembrane tyrosine kinase. AREG is expressed in various tissues, including the reproductive and urinary systems, circulatory system and respiratory and gastrointestinal tracts (14). AREG overexpression provides self-sufficient growth and survival signals in lung carcinoma cells (15). AREG up-regulation has been associated with resistance to chemotherapy, such as doxorubicin and cisplatin in liver cancer cells $(16,17)$ and cisplatin in breast cancer cells (18). AREG inhibits EGFR tyrosine kinase inhibitor (TKI)-induced apoptosis in NSCLC cells (15).

The tumor suppressor p53 trans-activates numerous target genes in response to DNA damage (19). Comprehensive expression analyses showed that AREG was a direct target of Ser46 phosphorylated p53 (19). In contrast, AMR is a synthetic anthracycline derivative anticancer agent that inhibits DNA topoisomerase II (6). Therefore, we inferred that AMR causes DNA damage and consequently up-regulates the expression of AREG via Ser46-phosphorylated p53.

The EGFR pathway has not been studied in detail in SCLC and there are little data available regarding the presence of EGFR in SCLC tissue (20). Previous studies have shown that EGFR, p-AKT and p-ERK expressions were detected in $37 \%, 24 \%$ and $13 \%$ of tumor specimens, respectively, in 107 patients who underwent surgery for SCLC (21). Furthermore, it was reported that an EGFR TKI, as well as a monoclonal EGFR antibody, showed anticancer activity against SCLC cell lines (20, 22). However, EGFR TKIs showed no significant clinical benefits in a phase II clinical trial in patients with SCLC (23). Indeed, the response to monoclonal EGFR antibodies can be predicted by the AREG secretion in gastric cancer cell lines (24).

In conclusion, up-regulation of AREG is induced by exposure to AMR-OH and confers resistance to the agent in AMR-OH-resistant cells. The present results support the clinical evaluation of CET/AMR in AMR-resistant lung cancer patients.

\section{Acknowledgements}

We thank Mr. Kei Kunimasa for his technical support and productive discussion. This work was supported by JSPS KAKENHI 25461158 and Research Grants from Daiichi Sankyo Co., LTD. to Motoko Tachihara and Research Grants from Astellas Pharma Inc., as well as Ono Pharma Co., LTD. to Yoshihiro Nishimura.

\section{References}

1 Siegel R, Naishadham D and Jemal A: Cancer statistics, 2012. CA Cancer J Clin 62: 10-29, 2012.

2 Shepherd FA, Crowley J, Van Houtte P, Postmus PE, Carney D, Chansky K, Shaikh Z and Goldstraw P: The International Association for the Study of Lung Cancer lung cancer staging project: Proposals regarding the clinical staging of small cell lung cancer in the forthcoming (seventh) edition of the tumor, node, metastasis classification for lung cancer. J Thorac Oncol 2: 1067-1077, 2007.

3 Takakuwa O, Oguri T, Ozasa H, Uemura T, Kasai D, Miyazaki $\mathrm{M}$, Maeno $\mathrm{K}$ and Sato S: Overexpression of MDR1 in amrubicinol-resistant lung cancer cells. Cancer Chemother Pharmacol 68: 669-676, 2011.

4 Knez L, Sodja E, Kern I, Košnik M and Cufer T: Predictive value of multidrug resistance proteins, topoisomerases II and ERCC1 in small cell lung cancer: A systematic review. Lung Cancer 72: 271-279, 2011.

5 Yip KW and Reed JC: Bcl-2 family proteins and cancer. Oncogene 27: 6398-6406, 2008.

6 Horita N, Yamamoto M, Sato T, Tsukahara T, Nagakura H, Tashiro K, Shibata Y, Watanabe H, Nagai K, Nakashima K, Ushio R, Ikeda M, Kobayashi N, Shinkai M, Kudo M and Kaneko T: Amrubicin for relapsed small-cell lung cancer: A systematic review and meta-analysis of 803 patients. Sci Rep 6: 18999, 2016.

7 Kurata T, Okamoto I, Tamura K and Fukuoka M: Amrubicin for non-small-cell lung cancer and small-cell lung cancer. Invest New Drugs 25: 499-504, 2007.

8 Kaira K, Sunaga N, Tomizawa Y, Yanagitani N, Shimizu K, Imai H, Utsugi M, Iwasaki Y, Iijima H, Tsurumaki H, Yoshii A, Fueki N, Hisada T, Ishizuka T, Saito R and Mori M: A phase II study of amrubicin, a synthetic 9-aminoanthracycline, in patients with previously treated lung cancer. Lung Cancer 69: 99-104, 2010.

9 Kim YH, Ishii G, Goto K, Ota S, Kubota K, Murata Y, Mishima M, Saijo N, Nishiwaki Y and Ochiai A: Expression of breast cancer resistance protein is associated with a poor clinical outcome in patients with small-cell lung cancer. Lung Cancer 65: 105-111, 2009

10 Tabara K, Kanda R, Sonoda K, Kubo T, Murakami Y, Kawahara A, Azuma K, Abe H, Kage M, Yoshinaga A, Tahira T, Hayashi K, Arao T, Nishio K, Rosell R, Kuwano M and Ono M: Loss of activating EGFR mutant gene contributes to acquired resistance to EGFR tyrosine kinase inhibitors in lung cancer cells. PLoS One 7: e41017, 2012.

11 Hatakeyama Y, Kobayashi K, Nagano T, Tamura D, Yamamoto M, Tachihara M, Kotani Y and Nishimura Y: Synergistic effects of pemetrexed and amrubicin in non-small cell lung cancer cell lines: Potential for combination therapy. Cancer Lett 343: 74-79, 2014.

12 Nagano T, Edamatsu H, Kobayashi K, Takenaka N, Yamamoto M, Sasaki N, Nishimura Y and Kataoka T: Phospholipase C $\varepsilon$, an effector of ras and rap small GTPases, is required for airway inflammatory response in a mouse model of bronchial asthma. PLoS One 9: e108373, 2014.

13 Yamane S, Ishida S, Hanamoto Y, Kumagai K, Masuda R, Tanaka K, Shiobara N, Yamane N, Mori T, Juji T, Fukui N, Itoh $\mathrm{T}$, Ochi $\mathrm{T}$ and Suzuki R: Proinflammatory role of amphiregulin, 
an epidermal growth factor family member whose expression is augmented in rheumatoid arthritis patients. J Inflamm 5: 5, 2008.

14 Berasain C and Avila MA: Amphiregulin. Semin Cell Dev Biol 28: 31-41, 2014.

15 Hurbin A, Dubrez L, Coll JL and Favrot MC: Inhibition of apoptosis by amphiregulin via an insulin-like growth factor-1 receptor-dependent pathway in non-small cell lung cancer cell lines. J Biol Chem 277: 49127-49133, 2002.

16 Castillo J, Erroba E, Perugorría MJ, Santamaría M, Lee DC, Prieto J, Avila MA and Berasain C: Amphiregulin contributes to the transformed phenotype of human hepatocellular carcinoma cells. Cancer Res 66: 6129-6138, 2006.

17 Chang CJ, Yin PH, Yang DM, Wang CH, Hung WY, Chi CW, Wei $\mathrm{YH}$ and Lee HC: Mitochondrial dysfunction-induced amphiregulin up-regulation mediateschemo-resistance and cell migration in HepG2 cells. Cell Mol Life Sci 66: 1755-1765, 2009.

18 Eckstein N, Servan K, Girard L, Cai D, Jonquieres GV, Jaehde U, Kassack MU, Gazdar AF, Minna JD and Royer HD: Epidermal growth factor receptor pathway analysis identifies amphiregulin as a key factor for cisplatin resistance of human breast cancer cells. J Biol Chem 283: 739-750, 2008.

19 Taira N, Yamaguchi T, Kimura J, Lu ZG, Fukuda S, Higashiyama $\mathrm{S}$, Ono $\mathrm{M}$ and Yoshida $\mathrm{K}$ : Induction of amphiregulin by $\mathrm{p} 53$ promotes apoptosis via control of microRNA biogenesis in response to DNA damage. Proc Natl Acad Sci USA 111: 717-722, 2014.
20 Fischer B, Marinov M and Arcaro A: Targeting receptor tyrosine kinase signalling in small cell lung cancer (SCLC): What have we learned so far? Cancer Treat Rev 33: 391-406, 2007.

21 Schmid K, Horvath ZB, Berger W, Haitel A, Cejka D, Werzowa J, Filipits M, Herberger B, Hayden H and Sieghart W: Dual inhibition of EGFR and mTOR pathways in small cell lung cancer. Br J Cancer 103: 622-628, 2010.

22 Tanno S, Ohsaki Y, Nakanishi K, Toyoshima E and Kikuchi K: Small cell lung cancer cells express EGFR and tyrosine phosphorylation of EGFR is inhibited by gefitinib ('Iressa', ZD1839). Oncol Rep 12: 1053-1057, 2004.

23 Moore AM, Einhorn LH, Estes D, Govindan R, Axelson J, Vinson J, Breen TE, Yu M and Hanna NH: Gefitinib in patients with chemo-sensitive and chemo-refractory relapsed small cell cancers: A Hoosier Oncology Group phase II trial. Lung Cancer 52: 93-97, 2006.

24 Kneissl J, Keller S, Lorber T, Heindl S, Keller G, Drexler I, Hapfelmeier A, Höfler $\mathrm{H}$ and Luber B: Association of amphiregulin with the cetuximab sensitivity of gastric cancer cell lines. Int J Oncol 41: 733-744, 2012.

Received March 22, 2017

Revised April 1, 2017

Accepted April 3, 2017 